\title{
Hep Par 1 Antibody Stain for the Differential Diagnosis of Hepatocellular Carcinoma: 676 Tumors Tested Using Tissue Microarrays and Conventional Tissue Sections
}

\author{
Zhen Fan, M.D., Matt van de Rijn, M.D., Ph.D., Kelli Montgomery, Robert V. Rouse, M.D. \\ Deparent of Pathology, Stanford University Medical Center, Stanford, California
}

\begin{abstract}
A well-characterized positive marker for hepatocellular differentiation would be a useful tool for the diagnosis of hepatocellular carcinoma (HCC). The recently commercially available Hep Par 1 antibody (clone OCH1E5.2.10) has been reported to be a sensitive marker for HCC in paraffin embedded sections. Of non-hepatocellular tumors, occasional carcinomas have been reported to stain, most fre-
\end{abstract} quently gastric adenocarcinomas. This study further evaluated the staining of this antibody on a large number of neoplasms using tissue microarray technology as well as conventional tissue sections. Six hundred seventy-six tumors, including 19 cases of HCC, were tested. Eighteen of 19 cases of HCC were positive, 3 showing $<5 \%$ staining. Two cases negative on the array showed focal staining when whole tissue sections from the same tumors were used. 16 of 34 cases of gastric carcinomas gave positive reactions, 4 of these showed less than $5 \%$ staining. Staining of gastric carcinomas was not limited to signet ring-type carcinomas or to areas of hepatoid differentiation. Only 1 of 11 cases of cholangiocarcinoma showed focal staining. We also noted several other tumors to stain occasionally, including adrenal cortical carcinoma (3/13), yolk sac tumor (2/9), colonic adenocarcinoma (8/106), lung carcinoma $(3 / 52)$, ovarian carcinoma $(5 / 48)$, and endocervical adenocarcinoma (1/5). We did not observe staining in pancreatic carcinoma (11), renal cell carcinoma (36), breast carcinoma (85), melanoma (25), or mesothelioma (5). This study supports Hep Par 1 as a useful marker in the differential diagnosis of HCC, but with significant limitations. Cautious use of this antibody in a panel with other positive (alpha fetoprotein, CD10, poly-

Copyright () 2003 by The United States and Canadian Academy of Pathology, Inc.

VOL. 16, NO. 2, P. 137, 2003 Printed in the U.S.A.

Date of acceptance: December 3, 2002.

Address reprint requests to: Robert V. Rouse, M.D., Department of Pathology L235, Stanford University Medical Center, 300 Pasteur Drive, Stanford, CA 94305-5324; fax: 650-725-7409; e-mail: rouse@stanford.edu. DOI: 10.1097/01.MP.0000052103.13730.20 clonal carcinoembryonic antigen) and negative (epithelial membrane antigen, monoclonal carcinoembryonic antigen, CD15) markers of hepatocellular differentiation may aid in the accurate diagnosis of HCC.

KEY WORDS: Hepatocellular carcinoma, Immunohistochemistry, Hepatocyte paraffin 1, Microarray. Mod Pathol 2003;16(2):137-144

The ability to distinguish hepatocellular carcinoma (HCC) from other malignancies by immunohistochemistry has been limited by the lack of a reliable positive marker for hepatocellular differentiation. Anti-alpha fetoprotein (anti-AFP) and antipolyclonal carcinoembryonic antigen (antiCEAp) antibodies are traditionally used positive markers for HCC. The sensitivity of AFP is low, ranging from $17-61.5 \%$ (1-4). AFP may also infrequently stain other types of carcinomas, including gastric, colonic, and cholangiocarcinomas (CC; $1-3$ ). Although quite specific for hepatocellular differentiation, canalicular staining with anti-CEAp or CD10 is reported to be present in $15-80 \%$ of HCC (4-7). The canalicular staining pattern of these antibodies is often difficult to interpret, thus limiting their use in the diagnosis of hepatocellular carcinoma.

The recently commercially available Hep Par 1 antibody (clone OCH1E5.2.10) stains normal and neoplastic hepatocytes. This antibody was developed in 1993 by Wennerberg et al. (8) using fixed liver as immunogen. The target antigen has not yet been fully determined. Several published studies have reported this antibody to be a sensitive marker for HCC $(2,4,8-12)$. Until very recently (4) it has been reported to be highly specific except for the staining of occasional gastric carcinomas and rare CCs. This study uses tissue microarray technology along with conventional tissue sections in the testing of a large number of tumors to further define the utility of Hep Par 1 in the differential diagnosis of HCC. 


\section{MATERIALS AND METHODS}

All the cases included in this study were obtained from the files at the Laboratory of Surgical Pathology at Stanford University Hospital. A total of 676 cases of malignancies, including 19 cases of HCC, were evaluated for Hep Par 1 staining.

\section{Tissue Microarrays}

The tissue microarrays were constructed using a tissue arrayer (Beecher Instruments, Silver Spring, MD) according to a previously described protocol $(13,14)$. Single $0.6-\mathrm{mm}$ cores of tissue taken from a representative area of each tumor were used to assemble the arrays. Five hundred eighty-eight tumors, including 14 HCCs, represented on three tissue arrays were evaluated for staining. Tissue validation was performed on HCCs that failed to react on the array by testing whole sections from the same cases.

\section{Tissue Sections}

Standard block tissue sections from 88 tumors, different from those on the arrays, were evaluated for Hep Par 1 staining (Table 1).

\section{Immunohistochemistry}

Five-micrometer-thick sections were cut from the microarray and conventional paraffin tissue blocks. The sections were deparafinized in xylene and rehydrated in graded series of ethanol. Heat epitope retrieval was performed by microwave boiling the deparaffinized sections in $10 \mathrm{mmol} / \mathrm{L}$ citrate buffer ( $\mathrm{pH}$ 6.0) for 12 minutes. The sections were then incubated with primary Hep Par 1 antibody for 30 minutes (clone OCH1E5.2.10, 1:80 dilution, DAKO, Carpinteria, CA). Staining was performed either manually, on a DAKO automatic stainer, or on a Ventana automated stainer using diaminobenzidine as the chromagen. Detection on the DAKO machine was made with EnVision+ (DAKO), a biotin-free detection system that consists of a secondary antibody covalently linked to peroxidase coated dextrose polymers. The Ventana stainer used a modified biotin-streptavidin-horseradish peroxidase detection method that may detect endogenous biotin $(17,18)$. A negative control for endogenous biotin staining using CD20 stain was performed in parallel with each Hep Par 1-stained slide. In all cases in which endogenous biotin was a problem, the stain was repeated with the EnVision + system. Positive external controls were used with cases lacking appropriate positive internal control.

\section{RESULTS}

Five hundred eighty-eight cases of tumor represented on tissue microarrays plus 88 cases of tumor represented on traditional tissue sections were evaluated for Hep Par 1 staining (Table 1). Hep Par 1 exhibits a granular cytoplasmic staining pattern. As the significance of staining in rare cells is difficult to determine, we considered $\geq 5 \%$ staining as being clearly significant but recorded cases with even rare positive cells as focal ( less than 5\%). Representative staining results are shown in Figures 1 and 2.

Hep Par 1 stained 18 of 19 cases of HCC. Three positively stained cases showed only very focal staining in less than $5 \%$ of the tumor. Two of these cases were initially negative on the array but demonstrated focal staining on repeat staining with whole sections taken from the same tumors. The completely negative case was a poorly differentiated HCC. The other two poorly differentiated cases showed $1 \%$ staining in one and $5-10 \%$ staining in the other. All 16 moderately differentiated cases were positive including two focally stained cases, each with less than 5\% staining. Hep Par 1 staining in HCC is frequently uneven and patchy in contrast to the more uniform staining of adjacent nonneoplastic liver. Both cases of hepatoblastoma stained uniformly and strongly. Of 3 cases of mixed HCC and CC, one case showed scattered staining in the hepatic component but not in the glandular component.

Of non-hepatocellular tumors, Hep Par 1 frequently stained gastric carcinomas. 16 of 34 (47\%) cases of gastric carcinoma stained; 12 (35\%) cases showed $5 \%$ or more staining. Eleven of 20 high grade or signet ring type carcinomas stained as compared with 5 of 14 cases of intermediate grade carcinoma. Four of the positive moderately differentiated cases showed $<5 \%$ staining. Gastric carcinomas frequently showed scattered and patchy staining, although several cases showed extensive staining in $>50 \%$ of the tumor. None of the gastric carcinomas showed hepatoid differentiation on the hematoxylin and eosin stains. A case of gastric neuroendocrine carcinoma was negative. We observed staining of metaplastic intestinal epithelium in the gastric mucosa without staining of the normal gastric epithelium.

Several other tumors also stained occasionally for Hep Par 1. Three of 13 adrenal cortical carcinomas stained. One case stained strongly and diffusely on whole tissue section. In addition, 2 of 9 cases of yolk sac tumors were positive. One case was a metastatic yolk sac tumor to the lung that showed $5 \%$ staining. The other case was a treated mixed germ cell tumor that showed staining in $20 \%$ of the residual yolk sac component. Neither positive yolk sac tumor con- 


\begin{tabular}{|c|c|c|c|c|}
\hline Tumor & Tissue Array & Whole Tissue Sections & $\begin{array}{c}\text { Combined } \\
\text { (Array }+ \text { Whole Section) }\end{array}$ & $\begin{array}{l}\text { Combined, } \\
\text { Cases with } \\
\geq 5 \% \text { Staining }\end{array}$ \\
\hline \multicolumn{5}{|l|}{ Hepatocellular carcinoma } \\
\hline Grade 2 & $12 / 14$ & $\wedge 4 / 4$ grade $2(2,<5 \%)$ & $16 / 16$ & $14 / 16$ \\
\hline Grade 3 & & $* 2 / 3$ grade $3(1,1 \%)$ & $2 / 3$ & $1 / 3$ \\
\hline Hepatoblastoma & $1 / 1$ & $1 / 1$ & $2 / 2$ & $2 / 2$ \\
\hline Cholangiocarcinoma & $0 / 1$ & $1 / 10$ & $1 / 11$ & $1 / 11$ \\
\hline $\begin{array}{c}\text { Mixed hepatocellular and } \\
\text { cholangiocarcinoma }\end{array}$ & ND & $1 / 3$ & $1 / 3$ & $1 / 3$ \\
\hline \multicolumn{5}{|l|}{ Gastric carcinoma } \\
\hline Grade 2 & ${ }^{*} 1 / 10(1,1-2 \%)$ & $* 4 / 4$ gr2 $(3,<5 \%)$ & $5 / 14$ & $1 / 14$ \\
\hline Grade 3 & $3 / 8$ & $3 / 4$ & $6 / 12$ & $6 / 12$ \\
\hline Signet ring & $2 / 5$ & $3 / 3$ & $5 / 8$ & $5 / 8$ \\
\hline Adrenal cortical carcinoma & $1 / 4$ & $2 / 9$ & $3 / 13$ & $3 / 13$ \\
\hline \multicolumn{5}{|l|}{ Lung carcinoma } \\
\hline Adenocarcinoma & $2 / 34$ & ND & $2 / 34$ & $2 / 34$ \\
\hline Squamous cell & $1 / 16$ & ND & $1 / 16$ & $1 / 16$ \\
\hline Non-small cell & $0 / 2$ & ND & $0 / 2$ & $0 / 2$ \\
\hline \multicolumn{5}{|l|}{ Colonic carcinoma } \\
\hline Adenocarcinoma & $2 / 83$ & $* 4 / 15(1,2 \%)$ & $6 / 98$ & $5 / 98$ \\
\hline Mucinous & $* 1 / 3(1,2 \%)$ & $1 / 3$ & $2 / 6$ & $1 / 6$ \\
\hline Signet ring & $0 / 2$ & ND & $0 / 2$ & $0 / 2$ \\
\hline Cloacogenic carcinoma & $0 / 2$ & ND & $0 / 2$ & $0 / 2$ \\
\hline \multicolumn{5}{|l|}{ Ovarian carcinoma } \\
\hline Clear cell & $* 2 / 6(1,5 \%)$ & $1 / 1$ & $3 / 7$ & $2 / 7$ \\
\hline Mucinous & $1 / 3$ & ${ }^{*} 1 / 3(1,1-2 \%)$ & $2 / 6$ & $1 / 6$ \\
\hline Serous & $0 / 27$ & ND & $0 / 27$ & $0 / 27$ \\
\hline Endometrioid & $0 / 7$ & ND & $0 / 7$ & $0 / 7$ \\
\hline Dysgerminoma & $0 / 1$ & ND & $0 / 1$ & $0 / 1$ \\
\hline \multicolumn{5}{|l|}{ Cervical carcinoma } \\
\hline Adenocarcinoma & $1 / 5$ & ND & $1 / 5$ & $1 / 5$ \\
\hline Squamous cell & $0 / 6$ & ND & $0 / 6$ & $0 / 6$ \\
\hline Prostatic carcinoma & $1 / 35$ & ND & $1 / 35$ & $1 / 35$ \\
\hline \multicolumn{5}{|l|}{ Esophageal carcinoma } \\
\hline Adenocarcinoma & ${ }^{*} 1 / 6(1,<1 \%)$ & ND & $1 / 6$ & $0 / 6$ \\
\hline Squamous & $0 / 7$ & ND & $0 / 7$ & $0 / 7$ \\
\hline \multicolumn{5}{|l|}{ Endometrial carcinoma } \\
\hline Endometrioid & $* 1 / 26(1,2 \%)$ & ND & $1 / 26$ & $0 / 26$ \\
\hline Clear cell/papillary & $0 / 4$ & $0 / 1$ & $0 / 5$ & $0 / 5$ \\
\hline Yolk sac & ND & $2 / 9$ & $2 / 9$ & $2 / 9$ \\
\hline \multicolumn{5}{|l|}{ Pancreatic carcinoma } \\
\hline Adenocarcinoma & $0 / 10$ & ND & $0 / 10$ & $0 / 10$ \\
\hline Anaplastic & $0 / 1$ & ND & $0 / 1$ & $0 / 1$ \\
\hline Gallbladder adenocarcinoma & $0 / 1$ & ND & $0 / 1$ & $0 / 1$ \\
\hline Transitional cell & $0 / 30$ & ND & $0 / 30$ & $0 / 30$ \\
\hline Renal cell carcinoma & $0 / 36$ & ND & $0 / 36$ & $0 / 36$ \\
\hline Breast adenocarcinoma & $0 / 74$ & $0 / 14$ & $0 / 85$ & $0 / 85$ \\
\hline Melanoma & $0 / 25$ & ND & $0 / 25$ & $0 / 25$ \\
\hline Neuroendocrine carcinoma & $0 / 7$ & $0 / 3$ & $0 / 10$ & $0 / 10$ \\
\hline
\end{tabular}

Tumors that tested negative on the arrays but not included in the table include ear, nose, and throat squamous carcinomas (29 cases), thyroid papillary carcinoma (19 cases), seminoma (12 cases), mesothelioma (5 cases), neuroblastoma (3 cases), nephroblastoma (3 cases), carcinoma of unknown primary (3 cases), endometrial stromal sarcoma (3 cases), skin basal cell carcinoma (2 cases), and one case each of the following: malignant mixed mullerian carcinoma, thyroid follicular carcinoma, skin paget disease, skin adnexal adenocarcinoma, skin squamous carcinoma, thymoma, solitary fibrous tumor.

$\wedge$ Includes 2 cases originally negative on the array but showed focal staining $(<5 \%)$ on full tissue sections. The adjacent columns to the right thus reflect a total of 19 cases of hepatocellular carcinoma tested in this study-14 cases from the array ( 2 of these were also stained using standard sections) and 5 other cases stained with standard sections only.

* Includes cases with $\leq 5 \%$ staining, (\#, \#\#) = cases with focal staining, percent focal staining.

$\mathrm{ND}=$ not performed.

tained morphologically recognizable hepatoid elements. One case of immature teratoma showed focal staining in the glandular component but the yolk sac component was negative. Other infrequently stained tumors include carcinomas of colon (8/106 adenocarcinoma), lung (2/34 adenocarcinoma, $1 / 16$ squamous cell), cervix $(1 / 5$ adenocarcinoma), ovary ( $3 / 7$ clear cell, $2 / 6 \mathrm{mu}-$ cinous), prostate (1/35), esophagus (adenocarcinoma $1 / 6)$, and endometrium (1/26 endometrioid carcinoma). Only 1 of 11 cases of cholangiocarcinoma stained very focally in $5 \%$ of the tumor.

Tumors that did not stain include pancreatic adenocarcinoma (11 cases), breast carcinoma (85 cases), renal cell carcinoma (36 cases), urinary tract transitional cell carcinoma (30 cases), ovarian serous carcinoma (27 cases), melanoma (25 cases), mesothelioma (5 cases), carcinoid/neuroendocrine carcinomas (10 cases), ear nose throat squamous cell carcinoma (29 cases), and papillary thyroid car- 

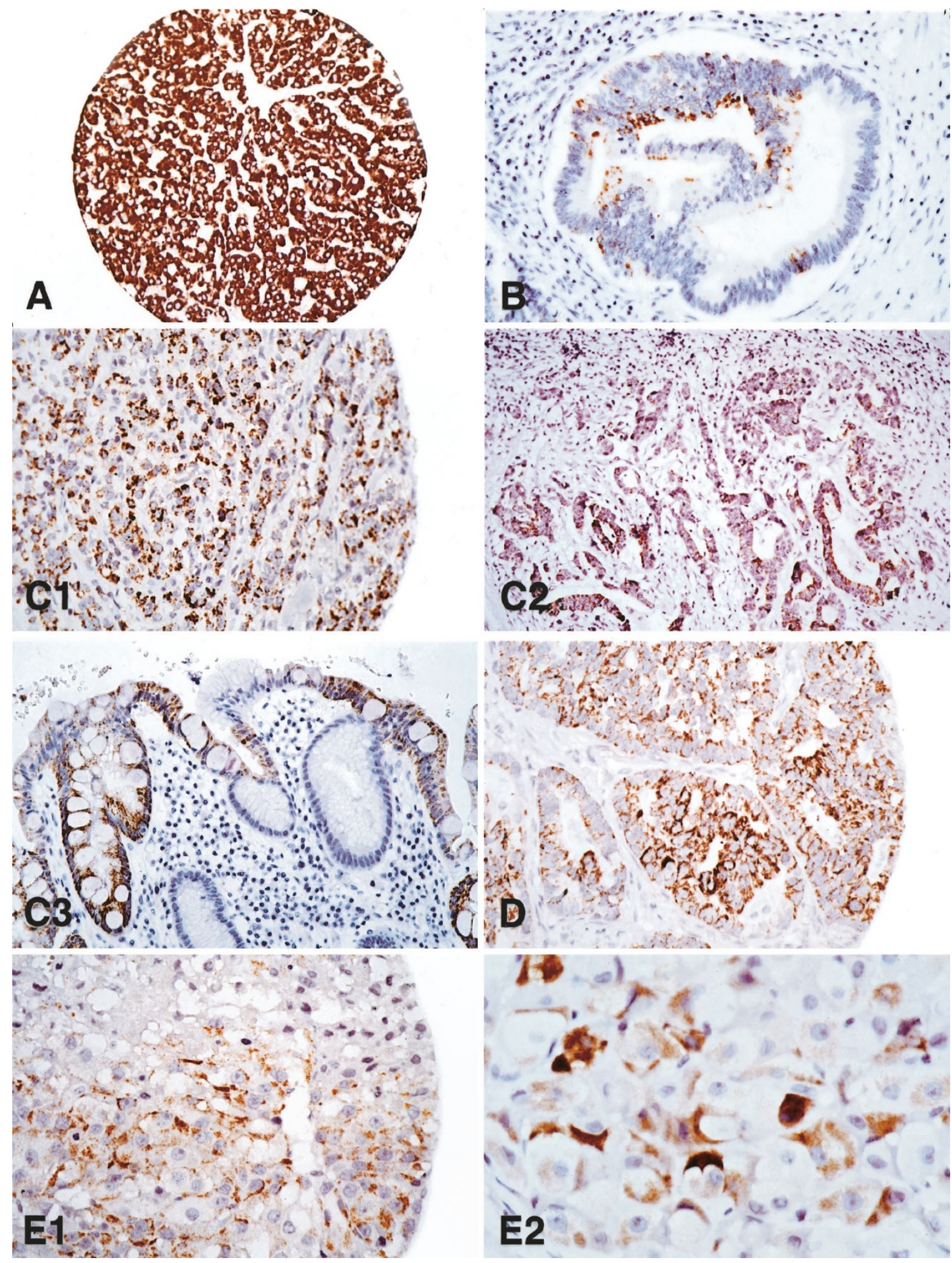

FIGURE 1. Hep Par 1 staining. A, hepatocellular carcinoma (array) shows strong confluent staining. B, cholangiocarcinoma (standard section) shows focal, faint staining. C, Section 1, gastric carcinoma, signet ring-type (array), with strong, diffuse cytoplasmic staining. Section 2, gastric carcinoma (standard section), Grade 3, with patchy staining. Section 3, staining of intestinal metaplasia in gastric epithelium and absence of staining in normal gastric epithelium (standard section). D, colonic adenocarcinoma (array) with strong staining. E, Sections 1 and 2, adrenal cortical carcinoma (array 1, standard section 2) shows patchy strong cytoplasmic staining 


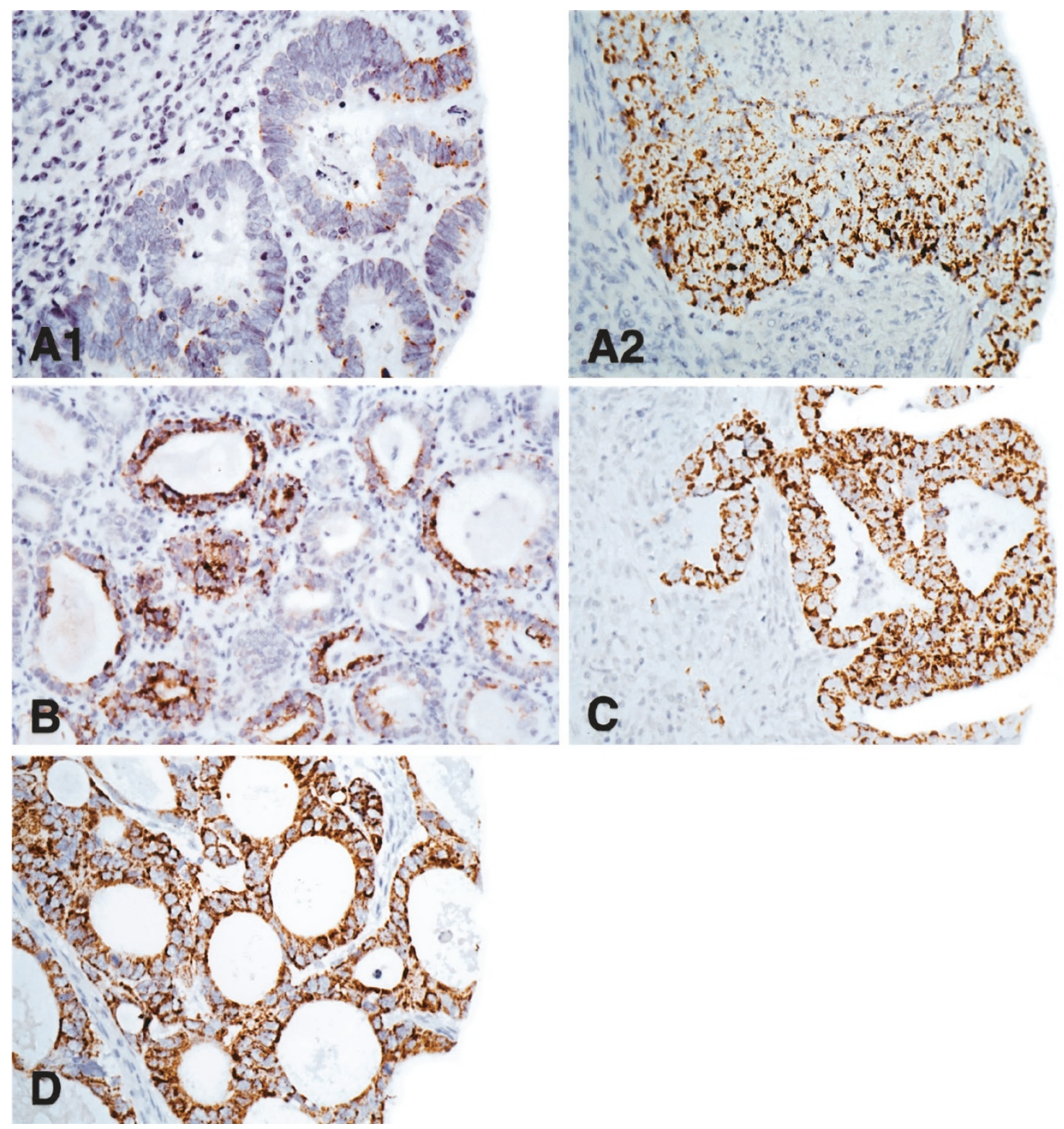

FIGURE 2. Hep Par 1 staining. A, Section 1, adenocarcinoma of lung (array) shows patchy staining. Section 2, squamous cell carcinoma of lung (array) shows strong staining. B, focal staining of glandular component in yolk sac tumor (standard section). C, endocervical adenocarcinoma (array) with strong, even staining. D, ovarian mucinous carcinoma (array) with strong, even staining.

cinoma (19 cases). Other unstained tumor types are listed in the footnote to Table 1.

\section{DISCUSSION}

Tissue microarray technology enabled us to test Hep Par 1 staining on a large number of tumors. The small core size, however, raises the possibility of false negative results due to non-representative sampling. On tumors for which we have both array and tissue section data, focally stained cases on tissue section, particularly with $<5 \%$ staining, are in some cases negative on the array. Recently, several studies have shown that two or more $0.6-\mathrm{mm}$ cores on tissue microarrays give a better representation than do single cores $(15,16)$. Tissue validation per- formed on two HCCs negative on the array showed focal staining (less than 5\%) in both tumors. In addition, a higher percentage of gastric carcinomas stained on tissue sections than on the arrays. This discrepancy is particularly evident in the moderately differentiated gastric carcinomas, nearly all of which showed very focal staining on whole sections. The array data correlated well with tissue section data on other tumors, including adrenal cortical, ovarian, and colonic carcinomas. Tissue arrays thus may be considered useful for establishing lower limits for the incidence of staining but may need to be supplemented with additional stained samples, especially for the evaluation of focal staining.

Endogenous biotin can be a significant source of false-positive staining in tissues such as liver and 
kidney after heat-induced epitope retrieval $(17,18)$. To avoid the problem of nonspecific endogenous biotin staining, we employed the EnVision+ detection system in the immunohistochemical staining procedure of our sections. EnVision+ is a biotinfree detection method that uses secondary antibody covalently linked to dextrose polymers coated with peroxidase molecules. Our sections detected with EnVision + resulted in clean background staining.
Hep Par 1 staining results from this study are compared with those published in the literature in Table 2 (2, 4, 8-12). Our results support Hep Par 1 as a sensitive marker of hepatocellular differentiation. Ninety-five percent (18/19) of HCCs showed staining at any level, versus $79 \%(15 / 19)$ when restricted to $>5 \%$ staining. Minervini et al. and Chu et al. $(2,4)$ observed that poorly differentiated HCCs are more likely to be negative for Hep Par 1 than are better differentiated cases (3/6 gr3 versus 9/10 gr2

TABLE 2. Hep Par1 Staining: Current Study Data Compared with Published Data

\begin{tabular}{|c|c|c|c|c|c|c|}
\hline \multirow{2}{*}{ Carcinoma Type } & \multicolumn{2}{|c|}{$\begin{array}{l}\text { Current Study Array } \\
\text { and Tissue }\end{array}$} & \multicolumn{2}{|c|}{$\begin{array}{l}\text { Other Studies Combined } \\
\qquad(2,4,8-12)\end{array}$} & \multicolumn{2}{|c|}{ Total Current and Other Studies } \\
\hline & $\begin{array}{l}\text { All Positive } \\
\text { Cases }\end{array}$ & $\begin{array}{c}\text { Cases with } \\
\geq 5 \% \\
\text { Staining }\end{array}$ & $\begin{array}{l}\text { All Positive } \\
\text { Cases }\end{array}$ & $\begin{array}{c}\text { Cases with } \\
\geq 5 \% \text { Staining }\end{array}$ & All Positive Cases & $\begin{array}{c}\text { Cases with } \\
\geq 5 \% \text { Staining }\end{array}$ \\
\hline Hepatocellular & 18/19 (95\%) & $15 / 19(79 \%)$ & $180 / 196(92 \%)$ & $170 / 196(87 \%)$ & $198 / 215(92 \%)$ & $185 / 215(86 \%)$ \\
\hline Hepatoblastoma & $2 / 2(100 \%)$ & $2 / 2(100 \%)$ & $13 / 13(100 \%)$ & $13 / 13(100 \%)$ & $15 / 15(100 \%)$ & $15 / 15(100 \%)$ \\
\hline Cholangiocarcinoma & $1 / 11(10 \%)$ & $1 / 11(10 \%)$ & $10 / 87(11 \%)$ & $5 / 87(6 \%)$ & $11 / 98(11 \%)$ & 6/98 (6\%) \\
\hline $\begin{array}{l}\text { Mixed hepatocellular and } \\
\text { cholangiocarcinoma }\end{array}$ & $1 / 3(33 \%)$ & $1 / 3(33 \%)$ & $5 / 8(63 \%)$ & $5 / 8(63 \%)$ & 6/11 (55\%) & $6 / 11(55 \%)$ \\
\hline Gastric & $16 / 34(47 \%)$ & $12 / 34(35 \%)$ & $11 / 31(35 \%)$ & $10 / 31(32 \%)$ & $27 / 65(42 \%)$ & $22 / 65(34 \%)$ \\
\hline Adrenal cortical & 3/13 (23\%) & $3 / 13(23 \%)$ & $0 / 10(0 \%)$ & $0 / 10(0 \%)$ & $3 / 23(13 \%)$ & $3 / 23(13 \%)$ \\
\hline \multicolumn{7}{|l|}{ Lung } \\
\hline Adenocarcinoma & $2 / 34(6 \%)$ & $2 / 34(6 \%)$ & $5 / 25(20 \%)$ & $5 / 25(20 \%)$ & $7 / 59(12 \%)$ & $7 / 59(12 \%)$ \\
\hline Squamous cell & $1 / 16(6 \%)$ & $1 / 16(6 \%)$ & $0 / 1(0 \%)$ & $0 / 1(0 \%)$ & $1 / 17(6 \%)$ & $1 / 17(6 \%)$ \\
\hline Colonic & $8 / 106(8 \%)$ & $6 / 106(6 \%)$ & $0 / 27(0 \%)$ & $0 / 27(0 \%)$ & $8 / 133(6 \%)$ & 6/133 (5\%) \\
\hline Cloacogenic & $0 / 2(0 \%)$ & $0 / 2(0 \%)$ & NA & NA & $0 / 2(0 \%)$ & $0 / 2(0 \%)$ \\
\hline \multicolumn{7}{|l|}{ Ovarian } \\
\hline Clear cell & $3 / 7$ (43\%) & $2 / 7$ (29\%) & $0 / 2(0 \%)$ & $0 / 2(0 \%)$ & $3 / 9(33 \%)$ & $1 / 9(11 \%)$ \\
\hline Mucinous & $2 / 6(33 \%)$ & $1 / 6(17 \%)$ & NA & NA & $2 / 6(33 \%)$ & $1 / 6(17 \%)$ \\
\hline Serous & $0 / 27(0 \%)$ & $0 / 27(0 \%)$ & NA & NA & $0 / 27(0 \%)$ & $0 / 27(0 \%)$ \\
\hline Endometrioid & $0 / 7(0 \%)$ & $0 / 7(0 \%)$ & NA & NA & $0 / 7(0 \%)$ & $0 / 7(0 \%)$ \\
\hline Unspecified & NA & NA & $4 / 24$ (17) & $4 / 24(17)$ & $4 / 24$ (17) & $4 / 24(17)$ \\
\hline Cervical adenocarcinoma & $1 / 11(9 \%)$ & $1 / 11(9 \%)$ & NA & NA & $1 / 11(9 \%)$ & $1 / 11(9 \%)$ \\
\hline Prostatic & $1 / 35(3 \%)$ & $1 / 35(3 \%)$ & $0 / 7(0 \%)$ & $0 / 7(0 \%)$ & $1 / 42(2 \%)$ & $1 / 42(2 \%)$ \\
\hline Yolk sac & $2 / 9(22 \%)$ & $2 / 9(22 \%)$ & $0 / 1(0 \%)$ & $0 / 1(0 \%)$ & $2 / 10(20 \%)$ & $2 / 10(20 \%)$ \\
\hline Seminoma & $0 / 12(0 \%)$ & $0 / 12(0 \%)$ & $0 / 4(0 \%)$ & $0 / 4(0 \%)$ & $0 / 16(0 \%)$ & $0 / 16(0 \%)$ \\
\hline \multicolumn{7}{|l|}{ Esophageal } \\
\hline Adenocarcinoma & $1 / 6(17 \%)$ & $0 / 6(0 \%)$ & NA & NA & $1 / 6(17 \%)$ & $0 / 6(0 \%)$ \\
\hline Squamous & $0 / 7(0 \%)$ & $0 / 7(0 \%)$ & NA & NA & $0 / 7(0 \%)$ & $0 / 7(0 \%)$ \\
\hline \multicolumn{7}{|l|}{ Endometrial } \\
\hline Endometrioid & $1 / 26(4 \%)$ & $0 / 26(0 \%)$ & $0 / 13(0 \%)$ & $0 / 13(0 \%)$ & $1 / 39(3 \%)$ & 0/39 (0\%) \\
\hline Papillary serous & $0 / 6(0 \%)$ & $0 / 6(0 \%)$ & $0 / 1(0 \%)$ & $0 / 1(0 \%)$ & $0 / 7(0 \%)$ & $0 / 7(0 \%)$ \\
\hline Pancreatic & $0 / 11(0 \%)$ & $0 / 11(0 \%)$ & $4 / 28(14 \%)$ & $1 / 28(4 \%)$ & $4 / 39(14 \%)$ & $1 / 39(3 \%)$ \\
\hline Gallbladder & $0 / 1(0 \%)$ & $0 / 1(0 \%)$ & $1 / 6(17 \%)$ & $1 / 6(17 \%)$ & $1 / 7(14 \%)$ & $1 / 7(14 \%)$ \\
\hline Transitional cell & $0 / 30(0 \%)$ & $0 / 30(0 \%)$ & $0 / 17(0 \%)$ & $0 / 17(0 \%)$ & $0 / 47(0 \%)$ & $0 / 47(0 \%)$ \\
\hline \multicolumn{7}{|l|}{ Thyroid } \\
\hline Follicular & $0 / 1(0 \%)$ & $0 / 1(0 \%)$ & $0 / 5(0 \%)$ & $0 / 5(0 \%)$ & $0 / 6(0 \%)$ & $0 / 6(0 \%)$ \\
\hline Papillary & $0 / 19(0 \%)$ & $0 / 19(0 \%)$ & $0 / 9(0 \%)$ & $0 / 9(0 \%)$ & $0 / 28(0 \%)$ & $0 / 28(0 \%)$ \\
\hline Skin, basal cell & $0 / 2(0 \%)$ & $0 / 2(0 \%)$ & $0 / 10(0 \%)$ & $0 / 10(0 \%)$ & $0 / 12(0 \%)$ & $0 / 12(0 \%)$ \\
\hline Skin and ENT squamous & $0 / 30(0 \%)$ & $0 / 30(0 \%)$ & $0 / 10(0 \%)$ & $0 / 10(0 \%)$ & $0 / 40(0 \%)$ & $0 / 40(0 \%)$ \\
\hline Thymoma & $0 / 1(0 \%)$ & $0 / 1(0 \%)$ & $0 / 8(0 \%)$ & $0 / 8(0 \%)$ & $0 / 9(0 \%)$ & $0 / 9(0 \%)$ \\
\hline Renal cell & $0 / 36(0 \%)$ & $0 / 36(0 \%)$ & $0 / 25(0 \%)$ & $0 / 25(0 \%)$ & $0 / 61(0 \%)$ & $0 / 61(0 \%)$ \\
\hline Breast & $0 / 85(0 \%)$ & $0 / 85(0 \%)$ & $0 / 20(0 \%)$ & $0 / 20(0 \%)$ & $0 / 105(0 \%)$ & $0 / 105(0 \%)$ \\
\hline Melanoma & $0 / 25(0 \%)$ & $0 / 251(0 \%)$ & $0 / 4(0 \%)$ & $0 / 4(0 \%)$ & $0 / 29(0 \%)$ & $0 / 29(0 \%)$ \\
\hline Mesothelioma & $0 / 5(0 \%)$ & $0 / 5(0 \%)$ & $0 / 19(0 \%)$ & $0 / 19(0 \%)$ & $0 / 24(0 \%)$ & $0 / 24(0 \%)$ \\
\hline Neuroendocrine & $0 / 7(0 \%)$ & $0 / 7(0 \%)$ & $5 / 76(7 \%)$ & $5 / 76(7 \%)$ & $5 / 83(6 \%)$ & $5 / 83(6 \%)$ \\
\hline
\end{tabular}

NA = no information available; ENT = ear, nose and throat.

Tumors that tested negative on the arrays but not included in the table include neuroblastoma ( 3 cases), nephroblastoma ( 3 cases), carcinoma of unknown primary ( 3 cases), endometrial stromal sarcoma (3 cases), and one case each of the following: malignant mixed mullerian carcinoma, skin paget disease, skin adnexal adenocarcinoma, solitary fibrous tumor. No information is available on these tumors in the literature for comparison.

Tumors reported to be negative in the literature but not tested in our study include and not included in the table above: carcinoma and metastatic carcinoma of unknown site (16 cases), vascular neoplasms (6 cases), small bowel carcinoma (11 cases), pheochromocytoma ( 2 cases), testicular embryonal carcinoma ( 7 cases), teratoma (6 cases) and choriocarcinoma (1 case), sarcoma ( 1 case), adenosarcoma ( 1 case), epithelioid sarcoma (10 cases), salivary gland tumors (19 cases) and 11 cases of undifferentiated carcinomas of various sites.

The neuroendocrine category included neuroendocrine carcinoma, small cell carcinoma, carcinoid, islet cell and Merkel cell carcinoma. The 5 positive cases reported in the literature were all of gastrointestinal or liver sites and comprised four neuroendocrine carcinomas and 1 carcinoid (4). 
versus $3 / 3$ grl stained), whereas other studies (9, 10) did not observe such a correlation. In this study, the only completely negative case was a poorly differentiated HCC. The other 2 poorly differentiated cases showed only focal staining. In contrast, all 16 moderately differentiated cases were positive, two of which showed less than $5 \%$ staining. These findings lend some support to the notion that poorly differentiated HCCs tend to lose reactivity for Hep Par 1. The heterogenous and sometimes focal nature of the staining in HCCs may lead to false negative results, particularly on small biopsy samples.

Although Hep Par 1 shows useful specificity for HCC in most instances, a notable exception is that it also frequently stains gastric carcinomas. We observed $47 \%(16 / 34)$ of gastric carcinomas to stain, and 35\% (12/34) when limited to cases with $5 \%$ or more staining. The poorly differentiated and signet ring tumor types stained more frequently and extensively than did the better differentiated types. Wennerberg et al. (8) tested 10 cases of gastric carcinomas, all high grade or signet ring type, and found 3 to stain. Recently, Maitra et al. (12) reported focal staining in five of six cases of gastric carcinomas with "hepatoid" differentiation. The staining in these tumors was ascribed to areas showing true hepatocellular differentiation, as evidenced by concurrent positivity for AFP and CEAp. Chu et al. (4) found staining in 2 of 12 conventional gastric adenocarcinomas, one of which was signet ring type, and in both of 2 carcinomas with hepatoid differentiation. Our cases of gastric carcinomas did not show "hepatoid" morphologic features, as described by Maitra et al. (12) and Chu et al. (4). The results of our study emphasize the frequent Hep Par 1 staining of conventional gastric carcinomas, independent of "hepatoid" differentiation. This is a significant limitation as metastatic gastric adenocarcinoma is frequently in the differential diagnosis of a liver mass subjected to biopsy. We also observed staining of metaplastic intestinal epithelium in the gastric sections; this is not surprising as normal small intestinal epithelium has been reported to stain.

The recent report of Chu et al. (4) describes staining in 5/21 lung adenocarcinomas and 4/24 ovarian carcinomas, similar to our findings. We also occasionally found Hep Par 1 to stain several other tumors for which staining has not been previously reported. These include carcinomas of adrenal cortex, colon, endocervix, esophagus, prostate and endometrium. Certain of these may be considered significant, even though their frequency of staining is not high: lung because of its overall frequency, colon because of its overall frequency and its drainage pattern, and adrenal cortical carcinoma be- cause of its histologic similarity to HCC. Unlike our results of no findings of staining in neuroendocrine carcinomas, Chu et al. (4) found four of nine such cases to be positive.

Although Hep Par 1 is $100 \%$ sensitive for hepatoblastomas (11) and does not stain other pediatric tumors such as neuroblastoma or nephroblastoma, we find some yolk sac tumors to stain (2/9 cases). We tested yolk sac tumors as they, like hepatoblastomas, are AFP positive. Fasano et al. (11) reported negative Hep Par 1 staining results in five germ cell tumors but did not specify the subtypes of these tumors, whereas Chu et al. (4) found no staining in 14 cases tested.

In summary, this study supports Hep Par 1 as a useful marker for HCC. It is not, however, entirely specific, showing frequent staining in gastric carcinomas. Several other tumors also stain occasionally, including yolk sac tumors as well as carcinomas of adrenal cortex, lung, colon, and ovary. The use of Hep Par 1 in conjunction with other positive (i.e., AFP, CD10 and CEAp) and negative (i.e., epithelial membrane antigen, monoclonal CEA, CD15) markers of HCC is recommended in the differential diagnosis of HCC.

Acknowledgments: The authors thank Lourdes D. Villanueva and Michael Durnen for technical assistance and Sky Shiviah for photographic expertise.

\section{REFERENCES}

1. Hurlimann J, Gardiol D. Immunohistochemistry in the differential diagnosis of liver carcinomas. Am J Surg Pathol 1991;15:280-8.

2. Mivervini MI, Demetris AJ, Lee RG, Carr BI, Madariaga J, Nalesnik MA. Utilization of hepatocyte-specific antibody in the immunocytochemical evaluation of liver tumors. Mod Pathol 1997;10:686-92.

3. Thung SN, Gerber MA, Sarno E, Popper H. Distribution of 5 antigens in hepatocellular carcinoma. Lab Invest 1979;41: 101-5.

4. Chu PG, Ishizawa S, Wu E, Weiss LM. Hepatocyte antigen as a marker of hepatocellular carcinoma. An immunohistochemical comparison to carcinoembryonic antigen, CD10, and alpha-fetoprotein. Am J Surg Pathol 2002;26:978-88.

5. Balaton AJ, Nehama-Sibony M, Gotheil C. Distinction betweeen hepatocellular carcinoma, cholangiocarcinoma, and metastatic carcinoma based on immunohistochemical staining for carcinoembryonic antigen and for cytokeratin 19 on paraffin sections. J Pathol 1988;156:305-10.

6. Ferrandez-Izquierdo A, Llombart-Bosch A. Immunohistochemical characterization of 130 cases of primary hepatic carcinomas. Pathol Res Pract 1987;182:783-91.

7. Koelman IA, Nap M, Huitema S, Kroma RAF, Houthoff HJ. Hepatocellular carcinoma, adenoma, and focal nodular hyperplasia. Comparative histopathologic study with immunohistochemical parameters. Arch Pathol Lab Med 1986;110: 1035-40.

8. Wennerberg AE, Nalesnic MA, Coleman WB. Hepatocyte paraffin 1: a monoclonal antibody that reacts with hepato- 
cytes and can be used for differential diagnosis of hepatic tumors. Am J Pathol 1993;143:1050-4.

9. Leong AS, Sormunen RT, Tsui WM, Liew CT. Hep Par 1 and selected antibodies in the immunohistological distinction of hepatocellular carcinoma from cholangiocarcinoma, combined tumours and metastatic carcinoma. Histopathology 1998;33:318-24.

10. Murakata LA, Ishak KG, Nzeako UC. Clear cell carcinoma of the liver: a comparative immunohistochemical study with renal clear cell carcinoma. Mod Pathol 2001;13:874-81.

11. Fasano M, Theise ND, Nalesnik M, Goswami S, Garcia de Davila MT, Finegold MJ, et al. Immunohistochemical evaluation of hepatoblastomas with use of the hepatocyte-specific marker, hepatocyte paraffin 1, and the polyclonal anticarcinoembryonic antigen. Mod Pathol 1998;11:934-8.

12. Maitra A, Murakata LA, Albores-Saavedra J. Immunoreactivity for hepatocyte paraffin 1 antibody in hepatoid adenocarcinomas of the gastrointestinal tract. Am J Clin Pathol 2001; 115:689-94.

13. Kononen J, Bubendorf L, Kallioniemi A, Barlund M, Schraml $\mathrm{P}$, Leighton S, et al. Tissue microarrays for high-throughput molecular profiling of tumor specimens. Nat Med 1998;4: 844-7.

14. Natkunam Y, Warnke RA, Montgomery K, Falini B, van de Rijn M. Analysis of MUM1/IRF4 protein expression using tissue microarrays and immunohistochemistry. Mod Pathol 2001;14:686-94.

15. Camp RL, Charette LA, Rimm DL. Validation of tissue microarray technology in breast carcinoma. Lab Invest 2000; 80:1943-9.

16. Hoos A, Urist MJ, Stojadinovic A, Mastorides S, Dudas ME, Leung DH, et al. Validation of tissue microarrays for immunohistochemical profiling of cancer specimens using the example of human fibroblastic tumors. Am J Pathol 2001; 158:1245-51.

17. Fan Z, Clark V, Nagle RB. An evaluation of enzymatic and heat epitope retrieval methods for the immunohistochemical staining of the intermediate filaments. Appl Immunohistochem 1997;5:49-58.

18. Rodriguez-Soto J, Warnke RA, Rouse RV. Endogenous avidin-binding activity in paraffin-embedded tissue revealed after microwave treaent. Appl Immunohistochem 1997;5: $59-62$.

\section{Book Review}

\section{O'Leary TJ: Advanced Diagnostic Methods in Pathology: Principles, Practice, and Proto- cols, 540 pp, Philadelphia, W. B. Saunders, 2002 (\$149.00).}

As stated by Dr. O'Leary in the introduction, "this book was envisioned as a reference that would bring together current information on newer techniques in biology and medicine that find use in diagnostic pathology," and his aim was certainly well achieved with this publication.

The text is divided into three sections. The first section discusses some theoretical principles concerning oncogenes and tumor suppresser genes, as well as antigens. This section of the text contains a summary of the genetic and metabolic pathways that regulate cell growth, survival, and death, and includes current information concerning the biology of apoptosis. In addition, the section on antigens contains valuable charts documenting the percentage of specific entities demonstrating positivity for the select antigens discussed. The second section deals with methodology: immunohistochemical and in situ hybridization techniques, blotting techniques, and methods of amplification. The third section discusses how the methodologies are applied to the individual organ systems, with additional chapters on infectious diseases and cytopathology. In addition, several of the chapters are interspaced with appendices, which present some of the specific procedures to be followed in the laboratory as well as quality control issues, the theory and principles behind the specific procedures, potential hazards, troubleshooting, etc.

This is a multi-authored textbook (there are 19 contributors, including Dr. O'Leary); each chapter is well written, well organized, and informative. There is, however, some unevenness in the amount of material devoted to the individual organ systems (there are only $4^{1 / 2}$ pages devoted to the testes, bladder, and prostatewhile the section on the pulmonary system contains almost 39 pages), but each section is extensively referenced, with several of the chapters containing more than 500 citations!

Advanced Diagnostic Methods in Pathology provides an excellent, comprehensive review of the current state of the art. It is understandable, considering the explosive amount of new information that is becoming available to us on practically a daily basis, that some of the material that is presented in this book will soon be outdated. This does not in anyway detract from this excellent addition to the literature, and I am pleased to recommend this textbook to all pathologists who need to have a comprehensive understanding of "what is currently out there today."

\section{Larry I. Giltman \\ Veterans Administration Medical Center- Atlanta \\ Emory University School of Medicine Atlanta, Georgia}

